\title{
Phonocardiographic Study on the Acute Rheumatic Carditis
}

\author{
Shozo YOSHIMURA, M.D., * Jiro SUGAI, M.D., * \\ Shin'ichi MITSUYA, M.D., Jun'ichi AKATSUKA, M.D.,** \\ and Tadao TAKAHASHI, M.D.***
}

Repeated phonocardiographic examinations were made on 65 cases with initial attack of rheumatic fever. A total of 52 patients having cardiac manifestation were served as consecutive study. Authors present the phonocardiographic features in the early stage of rheumatic carditis correlating with the activity of the disease; in most instances significant murmurs, gallop thythm and abnormal splitting of pulmonic second sound are coincident in acute phase. Among significant. murmurs apical mid-diastolic murmur displays its reversibility. The $\mathrm{E} / \mathrm{M}$ at apex or ratio of the duration of electrical systole (QT-interval) to that of mechanical systole (I-II interval in apical Medium PCG) varies with the degree of rheumatic activity. Increasing value of this ratio is parallel with rheumatic process. From these results presence of rheumatic activity would cause flabby or feeble state of myocardium which may also be an important factor in generating significant murmurs as well as actual "valvulitis".

$\mathrm{T}$ HE most significant and often fatal manifestation of rheumatic fever is carditis. Its clinical recognition usually depends upon the detection of cardiac murmurs. But in general the evaluation of cardiac murmurs may be difficult, since they often occur in patients associated with tachycardia or co-exist as a functional murmur. Precise and objective cardiac findings and differentiation of various types and qualities of murmurs are essential for the diagnosis of active rheumatic carditis. Attempts to evaluate the clinical significance and to clarify the characteristics of cardiac murmurs in rheumatic fever by means of graphic method have been made during past 2 decades. Schwarzchild and Feltenstein" recorded the apical diastolic murmur due to acute valvulitis by phonocardiographic method in 1935.

Since Bouillaud's first description ${ }^{23}(1835,1840)$, the cardiac murmurs in rheumatic fever have been said to have changing and transient nature. Thus many arguments on the mechanism of the production of murmurs have also been discussed; presumably either acute valvulitis or myocardial

From the First Department of Internal Medicine, Jikeikai School of Medicine, Tokyo

* The First Department of Internal Medicine.

**: Department of Pediatrics.

*a*: Professor of Internal Medicine. 
damage or both (2 lesions) are responsible for the production of murmurs.

We present in this report phonocardiographic observation of 52 cases with initial attack of rheumatic carditis. The purpose of this study is to find the characteristics of phonocardiographic changes in early stage of this illness and to solve the problem of pathophysiology of the mechanical phenomenon in the rheumatic heart through correlations of the clinical data with those supplied by phonocardiographic and electrocardiographic findings.

\section{Materials and Methods}

Fifty-two children and adolescents who admitted to Tokyo Hospital because of rheumatic carditis were examined repeatedly. In addition to these cases, 13 patients whose manifestation was only migratory polyarthritis or chorea were also studied as a control group. A total of these 65 cases were presumed to be in their first rheumatic attack. During the observation, recovery from acute attack occurred in all patients with or without cardiac involvement but later 7 cases of carditis died of repeated reccurrences.

Sixty-five cases with rheumatic fever were divided into 3 groups according to their conditions obtained from clinical data. Group 1: patients with moderate rheumatic activity and under moderate limitation of physical activity or class III in functional capacity. Group 2: patients with slight rheumatic activity and under class II in functional capacity. Group 3: patients with slight to moderate rheumatic activity but without recognizable cardiac involvement (control group).

The rheumatic activity was estimated by data obtained from physical, laboratory, electrocardiographic and roentgenographic examinations. In most cases phonocardiograms were taken in series whenever there was a change in either rheumatic activity or auscultatory fiinings. In some cases sound tracings were made only at their acute and convalescent phase.

The sound tracings were recorded by means of the Multichannel Phonocardiograph having 5 distinct frequency characteristics (Low, Medium-low, Medium, Medium-high, and High ranges), with the patient in the supine position, holding the breath at expiration. Tracings were recorded subsequently from Low (No. 1) to High (No. 5) at a film speed of $10 \mathrm{~cm}$. per second (at times, $5 \mathrm{~cm}$. per second). Recording was made at the apex, pulmonic area, aortic area, tricuspid area and the fourth left sternal border. Lead II of electrocardiogram was recorded simultaneously. At the same time, 12 leads electrocardiogram and P-A roentgenogram of the chest were taken, and from the latter "cardio-thoracic ratio" was measured to determine the cardiac enlargement. On the electrocardiogram, prolongation of atrioventricular conduction, deformity in QRS-complex, deviation of ST-segment, change in T-wave and prolongation of QT-interval or increase in corrected QT (QTC) were analysed.

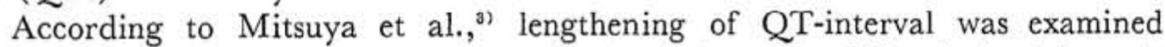
by comparing with the duration of the mechanical systole which obtained through following procedure: a distance between the beginning of central phase of the first heart sound and that of the second heart sound in apical "Medium" 


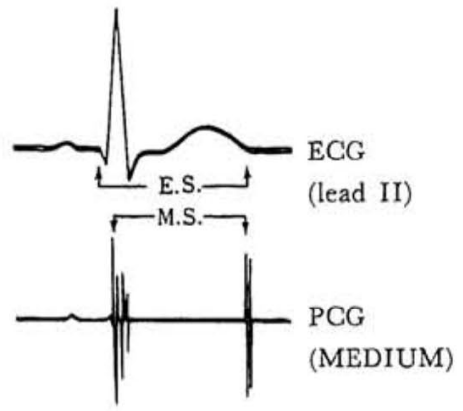

Fig. 1. Schema of phonocardiogram at apex.

E.S. : duration of electrical systole, M.S. : duration of mechanical systole.

phonocardiogram (Fig. 1). This ratio was tentatively expressed as "apical E/M" in percentage. Conditions affecting the duration of electrical systole other than rheumatic process such as digitalis effect, presence of congestive failure and pericarditis with effusion etc. were excluded from the calculation of "apical E/M". Duration of mechanical systole was not measured when there was a replacement of the first or second heart sound by murmur.

\section{Results}

Phonocardiographic findings in active carditis were very fluctuating or changing in nature.

I. Evolution of Abnormal Heart Sounds and Murmurs.

A. Abnormalities in Heart Sounds (Table I, Fig. 2, 3, 4)

In group 1 which included 43 patients, gallop rhythm occurred in 26 $(60.4 \%)$ and abnormal splitting of the second sound (split distance over $0.05 \mathrm{sec}$.) over pulmonic area in 30 (69.7\%) (Fig. 4). Group 2 consisted of 9 patients and showed similar incidence of gallop rhythm and split pulmonic second sound as group 1, but distinguished from group 1 in regard to the absence of presystolic gallop rhythm. In group 3, there were 13 patients,

Table I. Abnormalities in Heart Sounds

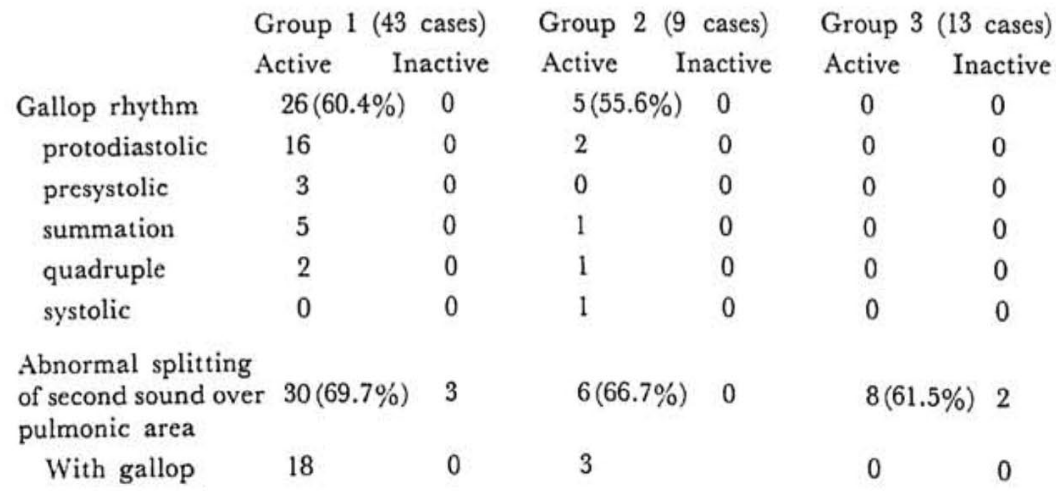


and split pulmonic second sound was the sole finding, showing the same incidence as in group 1 and 2. Among various types of gallop rhythm, protodiastolic gallop (Fig. 2) occurred most frequently, 18 out of 31 cases (58\%), and presystolic gallop occurred only in group 1.

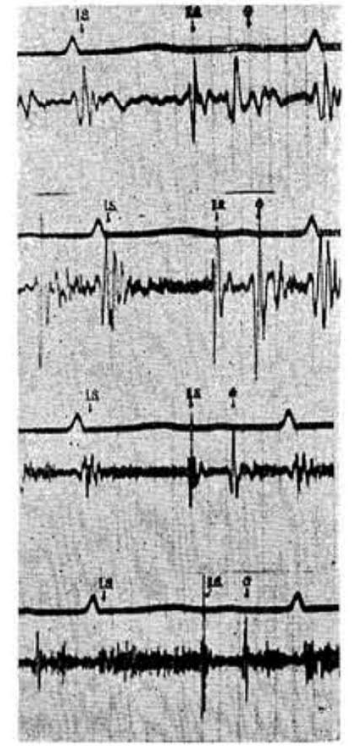

(a)

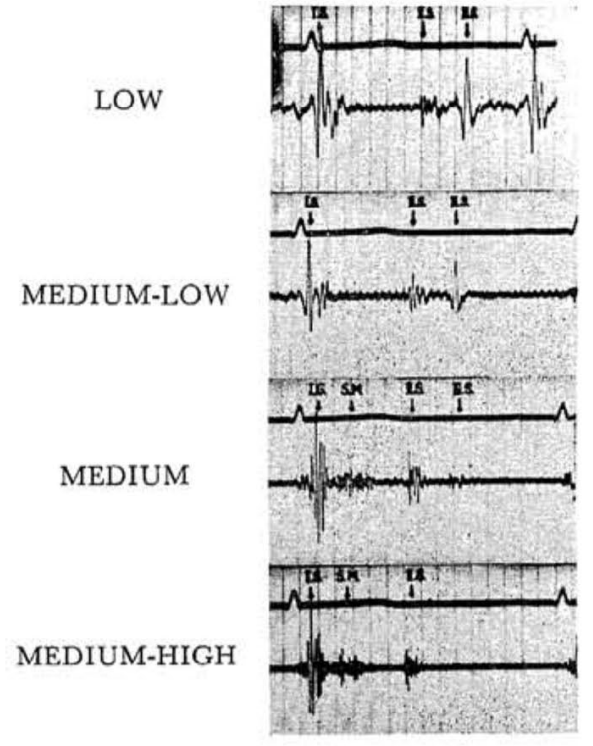

(b)

Fig. 2. Phonocardiogram at apex.

(a) A 12-year-old boy. Tracing was made at acute stage. High-pitched holosystolic murmur, loud 3rd sound (triple rhythm) and moderate diastolic murmur just followed after gallop sound.

(b) At convalescent stage. Disappearances of diastolic murmur, regress of 3rd sound and systolic murmur (only early and faint systolic murmur).

Electrocardiographically delayed $\mathrm{a}-\mathrm{v}$ conduction was seen in 15 out of 26 cases with gallop rhythm in group 1 and 4 out of 5 cases in group 2 . Increased QTC coincided with gallop rhythm less frequently, 10 in group 1 and 2 in group 2.

Moderate to marked enlargement of cardiac silhouette on X-ray examination was recognized in most cases with gallop rhythm. This condition was explained by increase in cardio-thoracic ratio and ranged from 0.50 to 0.78 with a mean of 0.58 .

When rheumatic activity was subsided, these abnormalities of heart sounds disappeared in the majority of cases (Table I, Fig. 2.).

B. Heart Murmurs (Table II, Fig. 2, 5, 6)

Configuration and type of heart murmurs over the different areas were also influenced by the rheumatic activity. In Table II, changes in type 
LOW
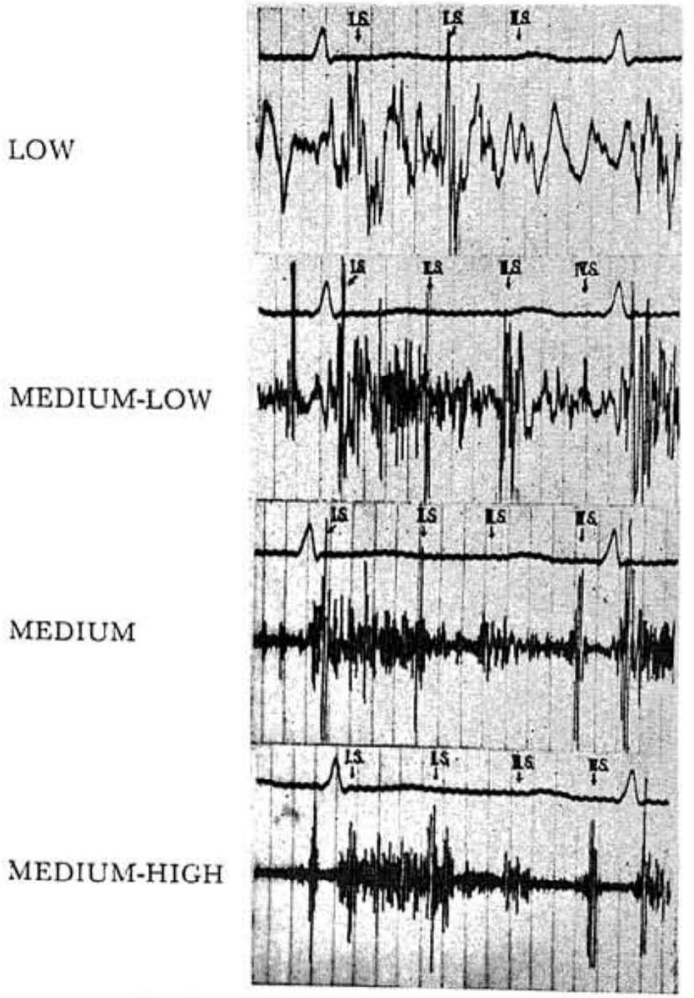

Fig. 3. Phonocardiogram at apex.

A girl aged 11. PCG shows high-pitched holosystolic murmur and quadruple rhythm.

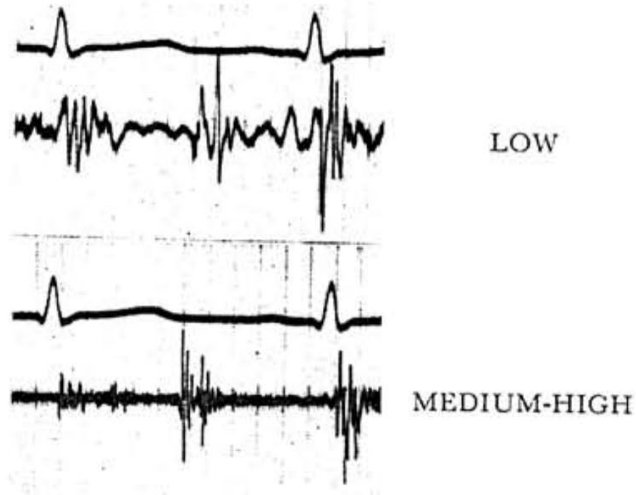

(a)
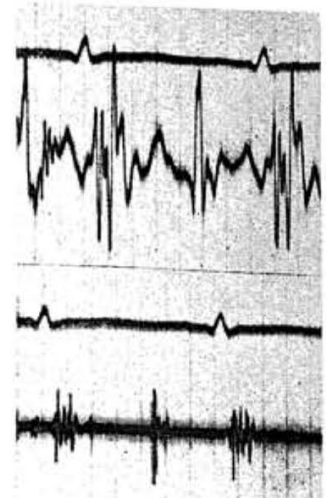

(b)

Fig. 4. Phonocardiogram over pulmonic area.

(a) In active phase. Distance of splitting 2nd sound is $0.05 \mathrm{sec}$.

(b) In inactive phase. Minimal splitting of 2 nd sound. 
Table II. Type and Configuration of the Murmurs over the Different Areas

\begin{tabular}{|c|c|c|c|c|}
\hline & \multicolumn{2}{|c|}{ Group 1 (43 cases) } & \multicolumn{2}{|c|}{ Group 2 (9 cases) } \\
\hline & Active & Inactive & Active & Inactive \\
\hline Apical systolic murmur & 43 & 39 & 9 & 6 \\
\hline holosystolic, harsh & 43 & 31 & 9 & 6 \\
\hline in decrescendo & 22 & 17 & 3 & 2 \\
\hline in crescendo & 9 & 7 & 2 & 2 \\
\hline in diamond shaped & 12 & 8 & 4 & 2 \\
\hline early or mid-systolic & 0 & 8 & 0 & 2 \\
\hline minimal or absent & 0 & 4 & 0 & 1 \\
\hline Apical diastolic murmur & 29 & 8 & 5 & 2 \\
\hline proto-diastolic & 5 & 4 & 0 & 0 \\
\hline mid-diastolic & 22 & 3 & 5 & 2 \\
\hline presystolic & 2 & 1 & 0 & 0 \\
\hline Basal diastolic murmur & 12 & 9 & 0 & 0 \\
\hline
\end{tabular}

LOW
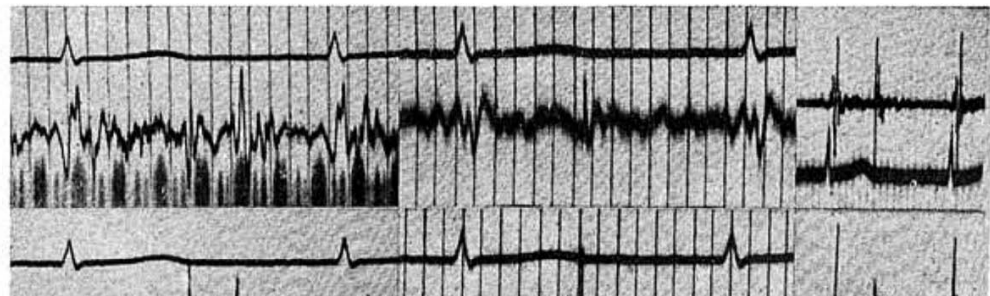

(n)
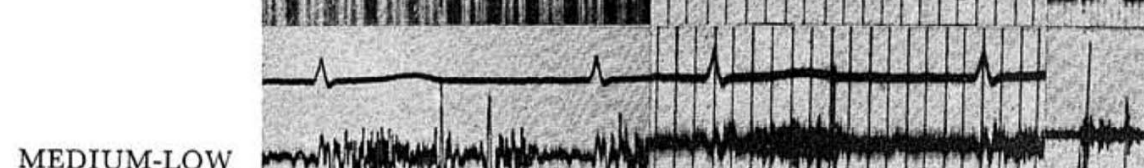

MEDIUM-LOW

MEDIUM

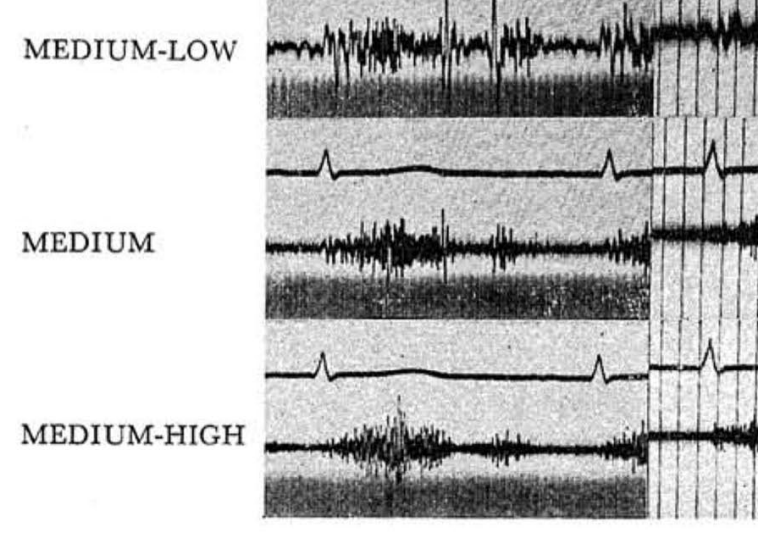

(a)

(b)

(c)

Fig. 5. Phonocardiogram at apex.

A girl aged 11. (a) 12 days after onset of illness. High-pitched diamond-shaped holosystolic murmur, loud 3rd sound and mid-diastolic murmur. (b) 2 months later. Loud 3rd sound and mid-diastolic murmur disappeared and systolic murmur altered its configuration to crescendo type. (c) 1 year later. Opening snap, a small vibration about $0.08 \mathrm{sec}$. after 2 nd sound. 


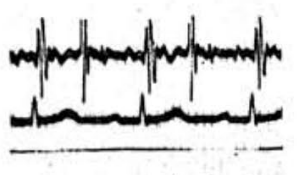

LOW

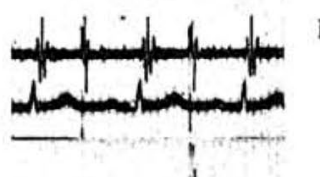

MEDIUM-LOW

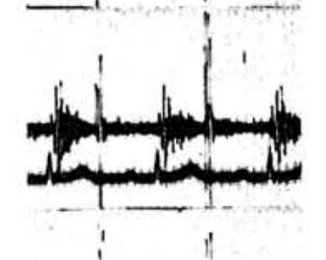

MEDIUM
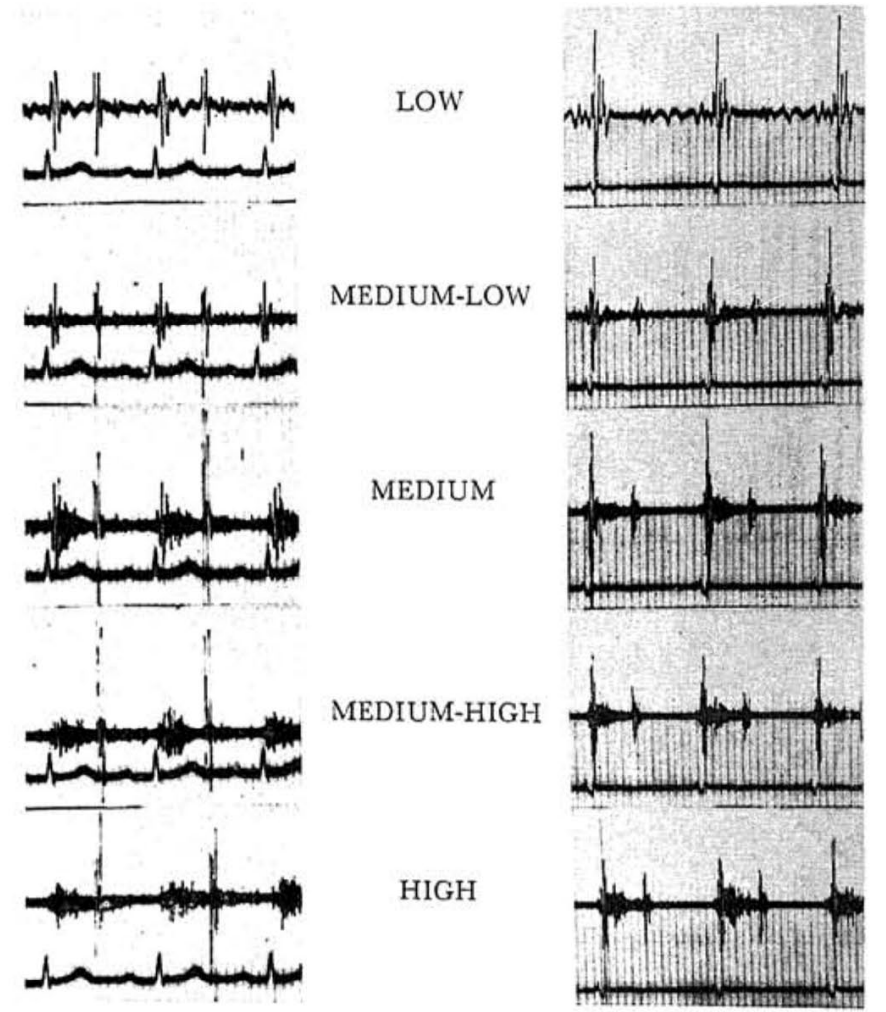

(a)

MEDIUM-HIGH

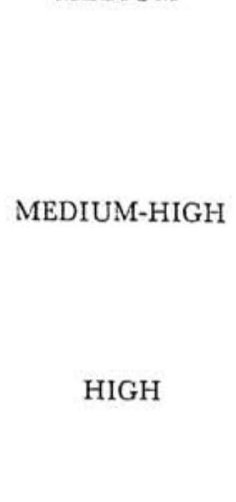

Fig. 6. Phonocardiogram over aortic area.

A boy, 12 years of age. (a) Diastolic murmur of grade 3 with relatively short duration in active phase. (b) 48 days later, activity subsided with simultaneous disappearance of diastolic murmur.

and configuration of the murmurs are presented. In both group 1 and 2 apical "holosystolic" murmurs have already been present in all instances at the first examination and its characteristics remained in about $74 \%$ and $67 \%$, respectively, after the subsidence of the rheumatic activity. Disappearance of holosystolic murmur was observed in only 4 cases and transformation to early or mid-systolic murmur in 8 cases (Fig. 2).

Apical diastolic murmur was present in 29 of group 1 and in 5 of group 2 at the first examination and co-existence of apical systolic murmur was seen in $86 \%$ of both groups. By regress of rheumatic process, 24 cases of those with apical diastolic murmur have lost their characteristic murmur. Particularly, mid-diastolic murmur disappeared most frequently $(22 / 27$, 81\%) (Fig. 2, 5).

Basal diastolic murmur was observed in only group 1 , and it disappeared in 5 (Fig. 6) and appeared in 2 after subsidence of the activity.

Disappearance of significant murmurs was concomitant with decrease 
in degree of the cardiac enlargement, cardio-thoracic ratio showed a marked diminution during this period.

II. Alteration of the apical E/M.

As previously reported, ${ }^{3}$ mean value of this ratio in normal children and adolescents lies $103 \pm 6$; in most cases with active rhcumatic carditis the value exceeds this level and is altered by degree of the rheumatic activity Fig. 7, 8

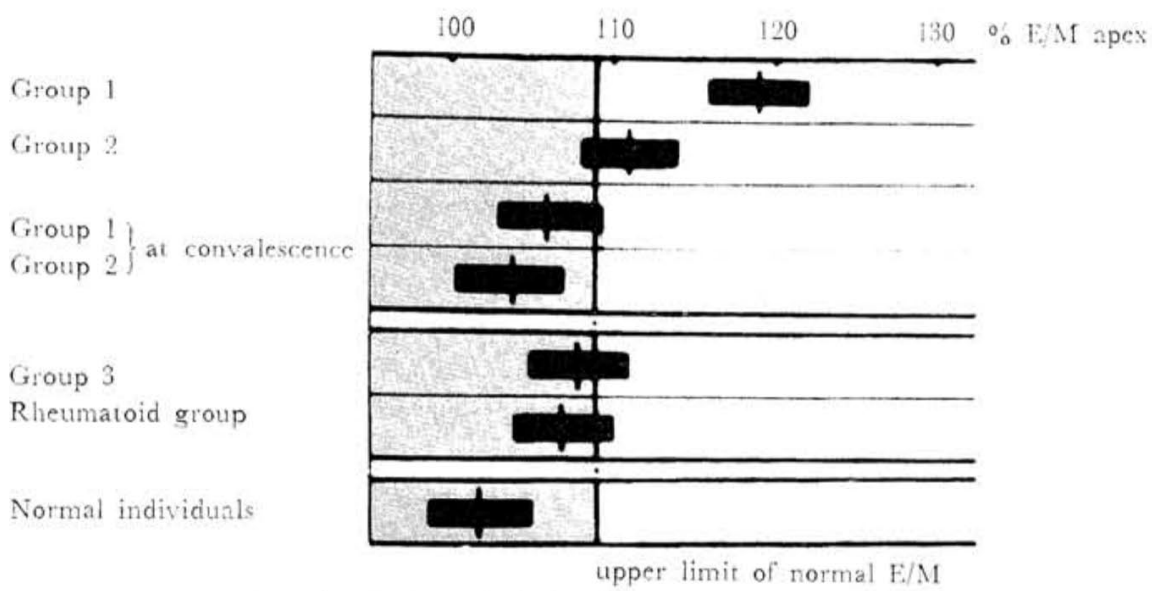

Fig. 7. Statistical distribution of apical E/M.

Delayed a-v conduction
Increased QTc
Increased apica! E/M
Abnormal splitting of 2nd
sound over pulmonic area

Group

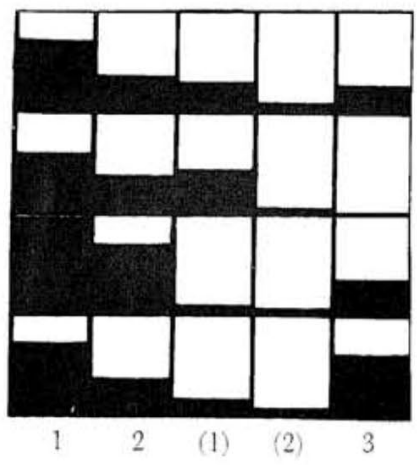

Fig. 8. Statistical distribution of clectro- and phonocardiographic findings. Black column indicates the occurrence of each items. (1), (2) at convalescent phase of group 1 and 2 respectively.

A. Apical E/M and Electrocardiographic and Phonocardiographic Findings (Table III)

Either lengthening of the electrical systole and/or shortening of the mechanical systole appeared to produce an increased apical E/M. This relationship is represented in Table III. In group I lengthening of the 
Table III. Factors in Increasing Value of Apical E/M

(A) Cases associated with prolonged QT-interval (24 cases).

\begin{tabular}{c|c|c|c|c|c|c}
\cline { 2 - 5 } & $\begin{array}{c}\text { No. of } \\
\text { patients }\end{array}$ & \multicolumn{2}{|c|}{$\mathrm{Q}^{-I^{*}>0.06^{\prime \prime}}$} & \multicolumn{2}{|c|}{ Q-I $\leqq 0.06^{\prime \prime}$} & Total \\
\cline { 2 - 6 } & QT-QII** $>0$ & QT-QII $\leqq 0$ & QT-QII $>0$ & QT-QII $\leqq 0$ & \\
\hline Group 1 & 43 & 8 & 4 & 6 & 1 & 19 \\
\hline Group 2 & 9 & 2 & 2 & 1 & 0 & 5 \\
\hline Group 3 & 12 & 0 & 0 & 0 & 0 & 0 \\
\hline Total & & 10 & 6 & 7 & 1 & 24
\end{tabular}

(B) Cases associated with normal QT-interval (31 cases).

\begin{tabular}{|c|c|c|c|c|c|c|}
\hline Group 1 & & 3 & 8 & 7 & 6 & 24 \\
\hline Group 2 & & 1 & 1 & 1 & 1 & 4 \\
\hline Group 3 & & 0 & 0 & 2 & 1 & 3 \\
\hline Total & 65 & 4 & 9 & 10 & 8 & 31 \\
\hline
\end{tabular}

Note:

* Q-I: Distance from the beginning of Q-wave on the electrocardiogram to that of the central phase of 1 st sound on the phonocardiogram at apex.

** $Q-I I$ : Distance from the beginning of $Q$-wave on the electrocardiogram to that of 2 nd sound on the phonocardiogram at apex.
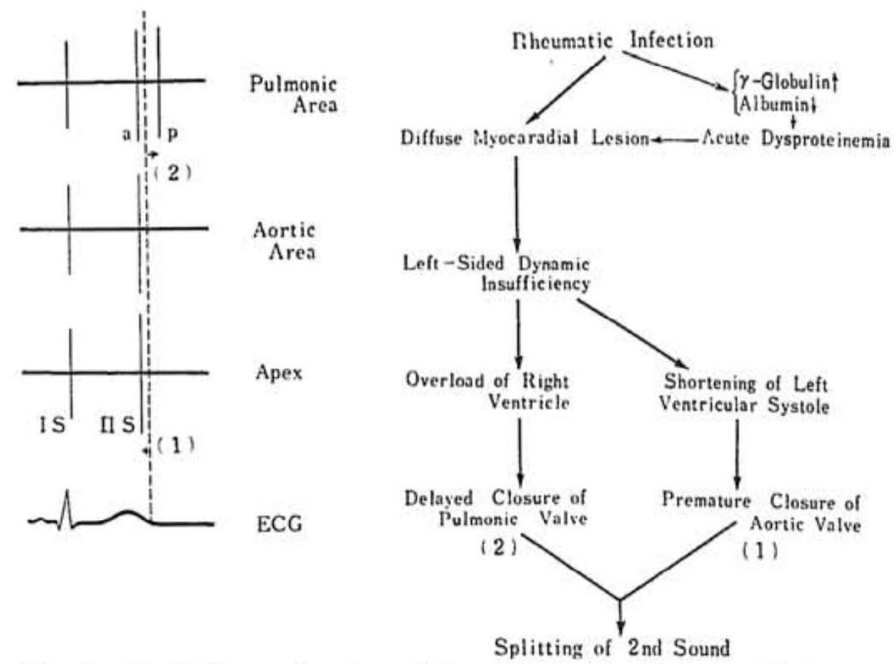

Fig. 9. Probable mechanism of the abnormal splitting of 2 nd sound over pulmonic area.

Left: Attitude of 2 nd sound over different areas. At apex, 2nd sound occurs before the completion of "repolarization" (dotted line) owing to mechanism (1). Over pulmonic area, "IIa" is coincident with 2nd sound at apex and aortic area, and "IIp" occurs fairly after the completion of repolarization owing to mechanism (2).

Right: Probable patho-physiological basis for occurrence of abnormal splitting of 2nd sound over pulmonic area. 
electrical systole seemingly resulted in a more marked increase in apical E/M. But in other items of this table significant difference could not be found out.

B. Apical E/M and Splitting of 2nd Sound over the Pulmonic Area

In Fig. 9, the mode of splitting is presented. The second sound over pulmonic area in rheumatic fever became split as a result of shortening of the ventricular mechanical systole in the left sided heart producing premature closure of the aortic valve occurring during the end of systole and delay in the closure of the pulmonic valve in the right side of the heart (Fig. 10).

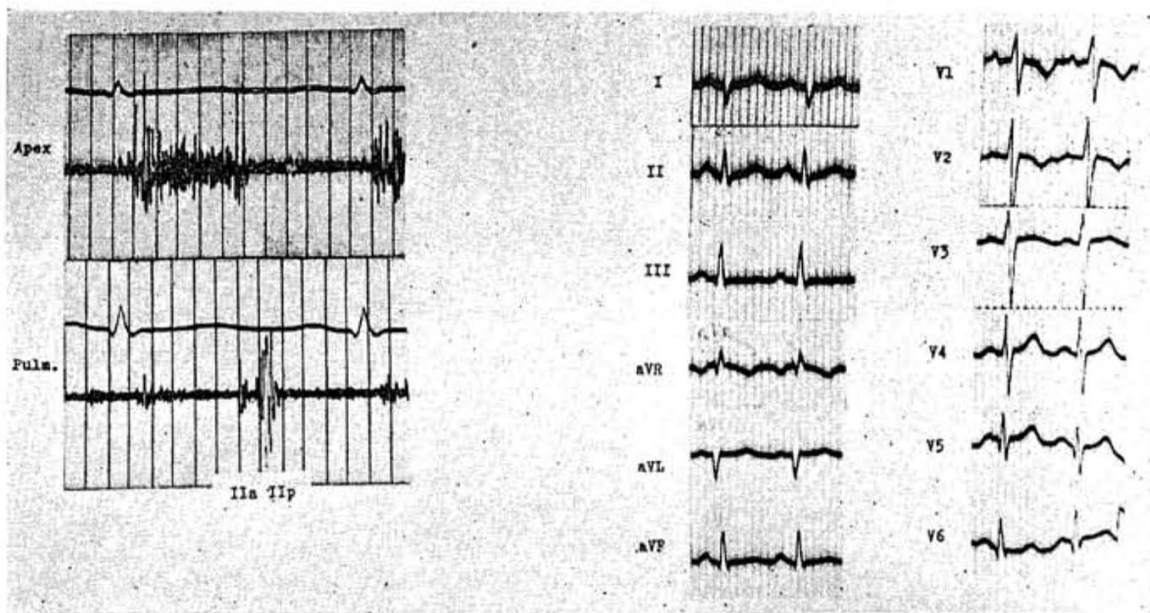

Fig. 10. Abnormal split of 2 nd sound over pulmonic area.

Left: Phonocardiogram at apex and over pulmonic area. Distance between 1 st and 2 nd sound at apex equal to distance between 1st and "pulmonic component" of 2 nd sound over pulmonic area. Apical E/M is $132 \%$. QTc 0.432 .

Right: Electrocardiogram shows slight ventricular hypertrophy.

\section{Discussion}

The most common and generally the earliest clinical signs of carditis in acute rheumatic fever are murmurs. In 1835, Bouillaud ${ }^{2}$ described at first auscultatory signs of a case with acute rheumatic fever. Since about a century ago many reports on the characteristics of the auscultatory data obtained from cases with acute rheumatic fever have been published.

Graphic registration of murmurs due to acute "valvulitis" was first performed by Schwarzchild and Feltenstein" in 1935. Since then advantage of phonocardiographic method for diagnosis of carditis has been recognized.

In our series apical systolic murmur had already been present in all cases at the first examination and it had high-pitched and holosystolic 
character. On the phonocardiogram, various types of configuration were observed as follows. Systolic murmur in decrescendo, which was characteristic of mitral regurgitation according to Luisada's description, ") was found in about one half of them and persisted throughout the course. In remaining one half, there were crescendo and diamond-shaped murmurs. The latter murmurs were relatively inconstant, and became minimal or disappeared in 4 instances.

Dual theories concerning the pathogenesis of apical systolic murmur have been suggested in the past. One of the theories originates on the basis of dilatation of left ventricle and mitral valve ring or mitral incompetency. ${ }^{(3-6), 8)-103}$ The other is based on improper closure of the mitral valve, which is due to inflammation and swelling of the mitral cusps. ${ }^{11}$ Luisada and Magri ${ }^{12)}$ considered that (1) dilatation of mitral ring, (2) edema and infiltration of the papillary muscle, and (3) edema of mitral leaflet may be responsible for the production of this murmur. Zilli and Gamna ${ }^{13)}$ have shown that apical systolic murmur was caused by the dilatation of mitral ring and stretching of papillary muscle in both early stage and established mitral lesion after disappearance of the activity. Bland and Jones $^{14}$ have pointed out that the apical high-pitched systolic murmur with long duration might be caused by an organic deformity of the mitral cusp if it became detectable after the disappearance of rheumatic activity. We agree with the opinion proposed by Zilli and Gamna. ${ }^{13)}$

The course of apical diastolic murmur was very interesting. In acute phase it was present in 34 cases and disappeared in 24 in convalescent phase. Especially the mid-diastolic murmur disappeared most frequently and rapidly. This particularity was first described by Coombs' and often entitled "Carey Coombs' Murmur". Bland and Jones" ${ }^{15}$ ) described this apical mid-diastolic murmur, if its intensity was minimal, was generated by the dilatation of the ventricle due to carditis and disappeared after a short duration. However, grade 3 to 4 mid-diastolic murmur if accompanied by systolic murmur was indicative of the deformity of mitral cusp. Luisada and Perez Montes, ${ }^{16)}$ Zilli and Gamna ${ }^{13)}$ pointed out that the mid-diastolic and presystolic murmur might be caused by an acute rheumatic carditis and would probably be due to "relative" mitral or tricuspid stenosis (dilatation of either venticle). Another theory concerning the cause of the diastolic murmur was described by Besterman ${ }^{17)}$ who states that a stiffened cusps and surface irregularities due to inflammatory process set up eddy current during rapid ventricular filling phase and cause the mitral diastolic murmur. Jones ${ }^{14}$ experienced a girl whose physical signs of carditis gradually disappeared after several years and died of an accident. The autopsy revealed no significant changes in her heart except "healed endocarditis." Garber ${ }^{18}$ ' reported the rheumatic heart disease without microscopic valvulitis and he postulated the cardiac murmurs (both systolic and 
diastolic) were probably due to myocarditis.

According to Massel, ${ }^{10}{ }^{17}$ the early inflammatory process is potentially reversible and the late fibrotic changes are not reversible. These facts might be reflected upon phonocardiographic findings!

The present study indicates that a rapid decrease in the cardio-thoracic ratio and apical E/M were evidently concomitant with the disappearance of apical diastolic murmur. Especially, mid-diastolic murmur disappeared in 22 out of $27(81.5 \%)$. Of course, there occurred actual stenosis of mitral orifice due to inflammatory process as mentioned by Besterman ${ }^{17}$, but we are of the opinion that the myocardial factor may play an important role for the development of the murmur.

Basal diastolic murmur was already present in 12 cases at the first examination. Its development to typical murmur of the aortic regurgitation occurred fairly later. But every case had high-pitched character and in 6 its duration reached to mid- to end-diastole. Delayed appearance was observed in 2 cases during the period of inactive phase. In 5 cases this type of murmur disappeared when rheumatic activity subsided, with decrease in degree of cardiac enlargement. In these cases, enlargement of the heart might be responsible for generating murmur to some extent.

As previously reported, there occurred significant discrepancy between electrical and mechanical phenomenon in rheumatic heart. Lengthening of the duration of electrical systole in rheumatic fever was noticed by Drawe et al. ${ }^{20}$ Taran and Szialgyi ${ }^{21}$ advocated that lengthening of the electrical systole was parallel with intensity of the rheumatic process. But such interpretation caused some discussion by Pokress and Goldberger. ${ }^{22}$ At any rate, diffuse myocardial lesion may cause the increased QT-interval.

As shown in Table III and Fig. 7 and 8, increased QTc was present in $24(44 \%)$ out of 54 cases associated with increased apical E/M. Among these 24 cases, 14 showed premature occurrence of second sound on the PCG. In 14 of 30 cases which had no prolongation of QT-interval, premature occurrence of second sound was seen before the completion of the ventricular repolarization.

Thus in 28 out of 55 cases, apparently shortened duration of the mechanical systole was revealed.

In rheumatic carditis, inevitable myocardial damage occurs in left ventricle to some extent, and it may be promoted by coexisting acute dysproteinemia produced by an increase in $r$-globulin and a decrease in albumin after rheumatic infection. ${ }^{28), 24)}$ Under these circumstances, leftsided dynamic insufficiency will become evident and as a consequence following 2 phenomena will occur: premature closure of aortic valve in left side and delayed closure of pulmonic valve in right side, the latter being introduced by the right ventricular overloading secondary to the left-sided dynamic insufficiency. This, in turn, produced splitting second sound over pulmonic area. 


\section{SUMMARY}

(1) Repeated phonocardiographic examinations are valuable for diagnosis, evaluation and the treatment of rheumatic fever.

(2) Early phonocardiographic changes characteristic for active carditis are as follows:

(a) High-pitched holosystolic murmur at the apex.

(b) Relatively short mid-diastolic murmur at the apex.

(c) Loud extra sound, especially 3rd sound. Frequent occurrence of gallop rhythm.

(d) Abnormal splitting of second sound over pulmonic area.

(3) Apical diastolic murmur is caused mainly by dilatation of the left ventricle with flabby myocardium and will disappear easily by treatment.

(4) Increasing value of apical $\mathrm{E} / \mathrm{M}$ is proportional to the rheumatic process.

(5) In the mechanism of the genesis of split pulmonic second sound, premature closure of aortic valve due to shortened mechanical systole of the left ventricle plays a certain role.

\section{REFERENCES}

1. Schwarzchild M. M. and Feltenstein, M. D.: Am. Heart J. 10: 453, 1934.

2. Bouilland, J.: Traité clinique des maladies du coeur, Paris, 1835 and Traité clinique de Rheumatisme, Paris, 1840, cited from Handbuch der inneren Medizin (vierte Auflage) VI/1, Springer, s. 908, 1954 and from 17).

3. a. Mitsuya, S., et al.: Jap. Circulation J. 23: 471, 1959.

b. Mitsuya, S.: Unpublished Observations.

4. Berliner, K.: Heart J. 7: 189, 1931.

5. Cheer. S. M. and Dieuade, F. R.: J. Clin. Invest. 10: 889, 1931.

6. Taran, L. M. and Ordorico, D.: Pediatrics 5: 947, 1950.

7. Luisada, A. A.: The Heart Beat, Paul B. Hoeber, New York, 1953.

8. Ash, R.: Am. Heart J. 36: 89, 1948.

9. Coombs, C.F.: Rheumatic Heart Disease (1st ed.), Bristol, 1924.

10. Levine, S. A.: Clinical Heart Disease (3d ed.), Saunders, Philadelphia, 1945.

11. Besterman, E. M. M. and Thomas, G.: Brit. Heart J. 15: 113, 1953.

12. Luisada, A.A. and Magri, G.: Am. J. Med. 15: 25, 1953.

13. Zilli, A. and Ganma, G.: Am. J. Med. 17: 775, 1954.

14. Bland, E. F. and Jones, T. D.: J. A. M. A. 107: 569, 1936.

15. Bland, E. F. and Jones, T. D.: Ann. Int. Med. 37: 1006, 1952.

16. Luisada, A. A. and Perez Montes, L.: Ann. Int. Med. 33: 56, 1950.

17. Besterman, E. M. M.: Brit. Heart J. 17: 360, 1955.

18. Garber, C. Z.: Am J. Path. 9: 443, 1933.

19. Massel, B. F.: Med. Clin. North Amer. 42: 1343, 1958. 
20. Drawe, C. E., et al.: Am. J. Dis. Child. 53 : 1470, 1937.

21. a. Taran, L. M. and Szialgyi, N.: Am. Heart J. 33: 14, 1947.

b. Taran, L. M. and Szialgyi, N.: Brit. Heart J. 13: 10, 1951.

22. Pokress, M. J. and Goldberger, E. : Am. Heart J. 38: 423, 1949.

23. Hartmann, F.: Z. Rheumaforsch. 11: 65, 1952.

24. Chaptal, J.: Arch. f. ped. 11: 1055, 1954. 